\title{
Alcohol and new onset atrial fibrillation: a case-contro study of a current series
}

\author{
PEKKA KOSKINEN, MARKKU KUPARI, HANNU LEINONEN, \\ KIMMO LUOMANMÄKI \\ From the First Department of Medicine, Helsinki University Central Hospital, Helsinki, Finland
}

SUMMARY The aetiological role of alcohol in new onset atrial fibrillation was evaluated in a of case-control study of 100 consecutive patients aged 21-64 years. Clinical examination, routine or diagnostic tests, and echocardiography revealed an underlying disease or other identifiable factor $\stackrel{\infty}{\circ}$ for atrial fibrillation in 65 patients (group 1); 35 patients had idiopathic atrial fibrillation (group o 2). The most common diseases associated with atrial fibrillation were ischaemic heart disease $(21 \%)$, hypertension $(13 \%)$, and cardiomyopathy $(8 \%)$. Data on alcohol consumption were obtained by interviewing the patients and their age and sex matched controls on admission. The mean daily alcohol intake of group 2 patients during the week preceding atrial fibrillation was significantly larger than that of either their controls or group 1 patients. Compared with controls . significantly more patients in both groups with atrial fibrillation had consumed alcohol within two days of the onset of the arrhythmia. Significantly more patients had onset of arrhythmia on Wednesday, Thursday, or Friday than on any other weekday, including patients with high alcohol intake.

This study establishes alcohol as an important precipitating factor for new onset atrial fibrillation.

Alcohol has recently been incriminated as a common precipitating factor for atrial fibrillation, ${ }^{1-3}$ and electrophysiological studies have verified its arrhythmogenicity in long term alcoholics. ${ }^{45}$ Not only habitual heavy drinkers seem to be liable to arrhythmias; even apparently healthy moderate or infrequent drinkers may suffer an episode of atrial fibrillation after an alcohol binge. ${ }^{6-8}$

The incidence of alcohol related arrhythmias is uncertain and may vary between populations with different alcohol cultures. All previous studies on the subject have been either retrospective surveys or isolated case reports. In one study, up to $47 \%$ of all new cases of atrial fibrillation in middle aged patients were thought to be caused by alcohol, ${ }^{1}$ whereas other reports have given rates from less than $30 \%{ }^{8}$ to $63 \%{ }^{2}$ of otherwise unexplainable atrial fibrillation.

We have recently began a series of studies to eval-

Requests for reprints to Dr Markku Kupari, Cardiovascular Laboratory, Helsinki University Central Hospital, SF-00290 Helsinki, Finland.

Accepted for publication 2 December 1986 uate the frequency and clinical importance of alcohol related arrhythmias in the emergency ward of our hospital. In the present report we describe the aetiological factors in 100 consecutive young and mid- $\frac{0}{0}$ dle aged patients with new onset atrial fibrillation and compare their drinking habits with those of a $\delta$ matched control patient population.

\section{Patients and methods}

The study was carried out between 1 January and 20 September 1985 in the emergency ward of Helsinki University Central Hospital. The hospital serves a O population of approximately 900000 and there are $\underset{\omega}{N}$ 1600-1900 patient visits every month to the emergency ward. During the study period 101 pa-ce tients aged $<65$ years were admitted for their first $\mathbb{D}$ documented episode of atrial fibrillation. These were? the patients that we studied.

\section{AETIOLOGICAL EVALUATION}

The patients were examined by a cardiologist on the $\mathbb{D}$ day of admission or the next morning, when sinus rhythm had been restored in almost all of them. The 
identification of cardiac or non-cardiac diseases was based on history and clinical examination complemented by chest $x$ rays, cross sectional and M mode echocardiographic examination (Irex System III echocardiograph), blood tests (Coulter autoanalyser), serum sodium and potassium (ion selective electrode), calcium (enzymatic or atomic absorption spectrophotometry), magnesium (atomic absorption spectrophotometry), aspartate and alanine aminotransferases (enzymatic), $\gamma$ glutamyl transpeptidase (enzymatic), creatine kinase isoenzymes (enzymatic and electrophoretic), thyroxine (radioimmunoassay), triiodothyronine uptake (radioimmunoassay and Sephadex uptake), and blood ethanol (enzymatic). Lung function was studied if this was needed to confirm or exclude the presence of pulmonary disease.

\section{ASSESSMENT OF DRINKING HABITS}

At admission every patient was questioned by one of us about the types and amounts of alcoholic beverages they had consumed each day during the week preceding the arrhythmia. The total amount of alcohol was recorded as grams of ethanol. The patients were also asked to estimate if this was more or less, or approximately the same they were accustomed to. If the patient was not aware of the time of onset of atrial fibrillation information about alcohol intake during the week before admission was collected. The patients were also asked the number of weeks they had been totally abstinent during the past year. Blood ethanol concentration on admission was not measured in all patients because the first laboratory tests were ordered by the attending physician and not one of the investigators. The smoking and coffee drinking habits of the patients and controls were also recorded.

\section{CONTROL PATIENTS}

After the case had been identified the diagnoses on emergency ward records were checked to find a suitable control patient. The control patient had to be of the same age ( \pm 10 years) and sex as the case and the diagnosis or symptom for hospital admission had to be one of an acute illness. The majority of the controls were found and interviewed on the same day as the cases, and the rest were found within two or three days. They too were questioned about their alcohol and coffee consumption and smoking habits in the week before admission. We did not select the controls totally randomly because hospital patients are not a representative sample of the population. By excluding patients admitted with conditions known to be alcohol related, such as acute pancreatitis or drug intoxications, and with severe chronic diseases, such as cancer, we tried to avoid overrepresentation
Table 1 The referral diagnoses or symptoms of the control patients

\begin{tabular}{ll}
\hline Diagnosis or symptom & No \\
\hline Acute asthma or bronchitis & 11 \\
Suspicion of deep venous thrombosis & $11^{\star}$ \\
Ureteral colic & 10 \\
Pneumothorax (2 traumatic, 7 spontaneous) & 9 \\
Miscellaneous acute infectious disease & 8 \\
Acute appendicitis & 7 \\
Pleuropneumonia & 7 \\
Erysipelas & 3 \\
Minor traumas & 3 \\
Headache & 3 \\
Hernias & 3 \\
Miscellaneous (wound infection, haematuria, & 23 \\
abdominal pain, vertigo) & \\
\hline ॠDeep venous thrombosis was confirmed by venography in eight \\
patients.
\end{tabular}
patients.

of heavy drinkers and abstainers, respectively, in our control population. We also excluded patients admitted for acute cardiac diseases because the present study is part of a large project designed to evaluate the role of alcohol in various cardiac emergencies. Table 1 lists the diagnoses and symptoms of the control patients.

\section{STATISTICAL ANALYSIS}

The statistical comparisons between cases and controls were performed by McNemar's test when there were only two outcomes and Stuart-Maxwell statistics ${ }^{9}$ when there were more. We also tested for a linear trend in the $(\log )$ risk of atrial fibrillation with increasing alcohol consumption. ${ }^{10}$ The atrial fibrillation groups were compared by the $\chi^{2}$ test and Student's $t$ test for independent data. The distribution of the onset of the arrhythmia between different weekdays was analysed by a $\chi^{2}$ test for goodness of fit.

\section{Results}

There were 19 women and 82 men (mean age 48 years (range 21-64)). The mean age of the controls was also 48 years. One patient, a male alcoholic, could not be examined and he was excluded from the analysis. Data on alcohol and coffee drinking and smoking were obtained in 98 patients; one patient died of diabetic coma and pneumonia before he could be interviewed and one patient admitted after an electrical shock did not answer the questions. Cross sectional echocardiograms were obtained in 95 patients; two patients refused echocardiography (an otherwise healthy 21 year old man and a male alcoholic with previous myocardial infarction) and one patient died of acute myocardial infarction before the 
Table 2 Diseases associated with new onset atrial fibrillation

No

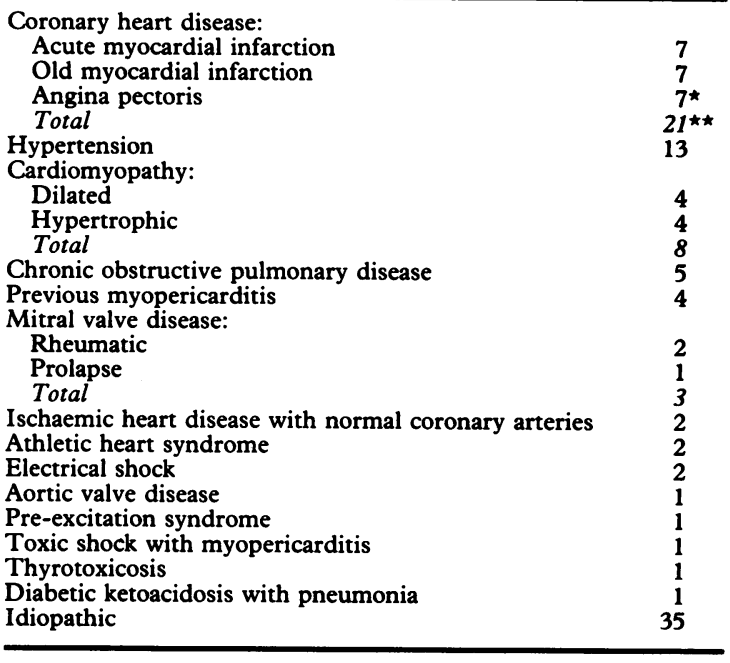

$\star 1$ patient also had pulmonary embolism; $\star \star 5$ patients also had hypertension

examination. Good quality echocardiographic recordings were obtained in 85 patients.

\section{DISEASES AETIOLOGICALLY ASSOCIATED WITH} ATRIAL FIBRILLATION

Sixty five patients (group 1) had a disease or other identifiable factor that could be aetiologically associated with atrial fibrillation (table 2). Twenty one of them had coronary heart disease; seven gave a history of typical effort related angina pectoris and 14 had acute or old myocardial infarction indicated by a history of prolonged chest pain and changes in the electrocardiogram or in serum enzymes or in left ventricular echocardiography. Hypertension was diagnosed in 18 patients; 16 were on antihypertensive treatment and two patients with previously undiagnosed hypertension had supine diastolic blood pressure $>110 \mathrm{~mm} \mathrm{Hg}$ in repeated measurements after admission. Five of the hypertensive patients also had coronary heart disease. Only four patients had hypertensive left ventricular disease (wall thickness $>14 \mathrm{~mm}$ on echocardiography). Dilated cardiomyopathy was diagnosed in four patients who had heart failure without other explanation and diffusely poor contraction of the left ventricle with an increased diastolic dimension (>60 mm) on echocardiography. Another four patients had the hypertrophic form of cardiomyopathy; the electrocardiogram showed increased left ventricular voltages in all, and the septal or free wall thickness exceeded $14 \mathrm{~mm}$ on echocardiography. Five patients had chronic obstructive pulmonary disease; they gave a long history of productive cough and dys- $c$. pnoea without heart disease and clinical exam- $\overrightarrow{\vec{F}}$ ination, chest $x$ rays, or lung function tests supported $\stackrel{?}{\circ}$ this diagnosis. Old myopericarditis was diagnosed in four patients and acute myopericarditis in one; all $\frac{\bar{c}}{\mathrm{c}}$. gave a history of a febrile illness (diphtheria in one, $\vec{\mathbb{}}$ rheumatic fever in one, acute toxic shock in one, $\frac{\Omega}{\sim}$ unspecified in two) and had localised or diffuse ab- $\omega$ normalities of myocardial contractions and peri- $\vec{O}$ cardial thickening or effusion on echocardiography. $\vec{\overrightarrow{ }}$ Significant valvar disease was found in four patients $\omega_{\sigma}$ (one rheumatic mitral stenosis, one rheumatic mitral regurgitation, one aortic regurgitation, and one mi- o tral valve prolapse). One of the patients had undergone cardiac catheterisation; in others the diagnosis was based on clinical and echocardiographic exam- $\infty$ inations. Athletic heart syndrome ${ }^{11}$ was diagnosed 0 in two patients; both had a long history of endurance training and an increased left ventricular end di- 3 astolic diameter (>56 mm) on echocardiography without impairment of left ventricular systolic func- $\vec{\theta}$ tion. Two patients had syndrome $X$ (ischaemic heart $\stackrel{\infty}{\checkmark}$ disease despite patent coronary arteries); both had previously undergone coronary angiographic and 0 perfusion studies. ${ }^{12}$ Other illnesses diagnosed in group 1 were thyrotoxicosis (one patient), preexcitation syndrome (one patient), and diabetic coma $\stackrel{\circ}{\Phi}$ with pneumonia (one patient). Two patients had $\varrho$ onset of arrhythmia after an electrical shock.

In 35 patients (six women and 29 men) we found no evidence of concomitant cardiac or non-cardiac disease (group 2).

DATA ON ALCOHOL CONSUMPTION

Nine patients (three women, six men) in group 1 and $\frac{7}{\sigma}$ 10 (five women, five men) controls had not drunk any alcohol during the past year. In group 2, five patients $\delta$ (three women, two men) and five controls (one $₹$ woman, four men) had been teetotallers for the 0 whole year. Table 3 shows the patients' and controls' estimates of their alcohol consumption for the week

Table 3 Estimation of alcohol consumption during the week before atrial fibrillation compared with normal use in cases and controls

\begin{tabular}{llllll}
\hline & \multicolumn{4}{l}{ Alcohol consumption } \\
\cline { 2 - 5 } & More & Less & The same & p \\
\hline All cases/controls & $17 / 9$ & $4 / 12$ & $77 / 77$ & $<0.05$ \\
Group 1/controls & $8 / 4$ & $2 / 9$ & $53 / 50$ & NS \\
Group 2/controls & $9 / 5$ & $2 / 3$ & $24 / 27$ & NS \\
\hline
\end{tabular}

Group 1, atrial fibrillation with an aetiologically associated disease.

Group 2, idiopathic atrial fibrillation. 


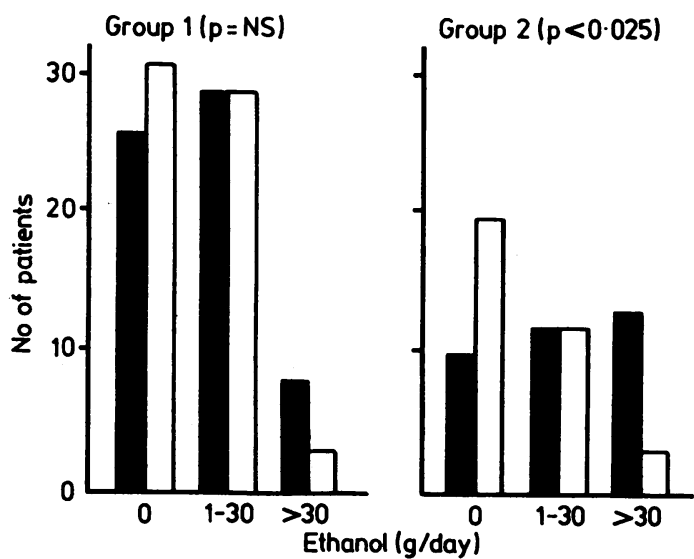

Fig 1 Average daily alcohol consumption of the patients (black columns) and their controls (white columns) during the week preceding the atrial fibrillation. Group 1, atrial fibrillation with an associated disease. Group 2, idiopathic atrial fibrillation.

preceding atrial fibrillation compared with their normal alcohol intake.

To analyse the data on the average amount of alcohol consumed daily during the week before atrial fibrillation we divided the patients and controls into three groups: ( $a$ ) abstainers; $(b)$ those with an average daily consumption of $1-30 \mathrm{~g}$ of ethanol; $(c)$ those with an average daily alcohol consumption of $>30 \mathrm{~g}$ of ethanol. Figure 1 shows the distribution of the patients and controls in these groups. Patients with atrial fibrillation had drunk more than the controls $\left(\chi^{2}=10.407 ; p<0.01\right)$. This difference came mainly from group 2 patients whose alcohol consumption had been significantly larger than that of their controls $\left(\chi^{2}=8.658 ; p<0.025\right)$ whereas the difference between patients in group 1 and their con-

Table 4 Frequency of abnormal laboratory findings in patients with new onset atrial fibrillation

\begin{tabular}{llrr}
\hline Variable & & Group 1 & Group 2 \\
\hline MCV & $>96 \mathrm{fl}$ & $6(57)$ & $1(29)$ \\
Serum potassium & $<3.7 \mathrm{mmol} / \mathrm{l}$ & $12(59)$ & $8(35)$ \\
& $<3.0 \mathrm{mmol} / 1$ & $1(59)$ & $2(35)$ \\
Serum calcium & $<2.25 \mathrm{mmol} / 1$ & $27(54)$ & $10(30)$ \\
& $<2.10 \mathrm{mmol} / 1$ & $3(54)$ & $2(30)$ \\
Serum magnesium & $>0.70 \mathrm{mmol} / 1$ & $5(51)$ & $3(27)$ \\
S-ASAT & $>40 \mathrm{IUU} / 1$ & $5(53)$ & $8(33)$ \\
S-ALAT & $>40 \mathrm{IUU} / 1$ & $10(54)$ & $10(32)$ \\
S- - -GT & $>50 \mathrm{IU} / 1$ & $18(53)$ & $8(31)$ \\
Blood ethanol & $>1 \mathrm{mmol} / \mathrm{l}$ & $5(12)$ & $7(17)$ \\
\hline
\end{tabular}

The figures in parentheses are the number of patients tested.

MCV, mean red cell corpuscular volume; S-ASAT, aspartate aminotransferase (serum); S-ALAT, alanine aminotransferase (serum); S- $\gamma$-GT, $\gamma$ glutamyl transpeptidase (serum).

Group 1, atrial fibrillation with an aetiologically associated disease. Group 2, idiopathic atrial fibrillation. trols was not statistically significant $\left(\chi^{2}=2.531\right.$; $\mathrm{p}=\mathrm{NS})$. The $(\log )$ risk of atrial fibrillation increased linearly with alcohol use in all cases $\left(\chi^{2}=8.880\right.$; $\mathrm{p}<0.025)$ and also in group $2\left(\chi^{2}=8.266\right.$; $\mathrm{p}<0.025$ ).

Seven of the patients with atrial fibrillation had used alcohol heavily ( $>75 \mathrm{~g}$ of ethanol daily) during the week preceding the arrhythmia: five of 35 in group 2 (vs none of their controls) and two of 63 in group 1 (vs one of their controls).

Alcohol use immediately before the arrhythmia was evaluated separately by our noting whether or not the cases and controls had drunk any alcohol during the two days preceding the arrhythmia or admission. There were 25 drinkers in group 1 (vs 13 controls; $\left.\chi^{2}=4.321, p<0.05\right)$ and 19 of 35 in group 2 (vs 4 controls; $\chi^{2}=11.529 ; p<0.001$ ). Twelve of the 29 patients whose blood ethanol concentration was measured on admission had been drinking recently. Their blood ethanol concentration was over $1 \mathrm{mmol} / \mathrm{l}$ (table 4).

\section{DATA ON COFFEE DRINKING AND SMOKING} Coffee drinking and smoking habits were not significantly different in cases and controls. In group $1,78 \%$ of cases and $83 \%$ of controls drank coffee daily and $35 \%$ of both cases and controls were current smokers. In group $2,77 \%$ of cases were coffee drinkers and $34 \%$ were smokers; the corresponding figures for the controls were $89 \%$ and $29 \%$.

\section{COMPARISONS BETWEEN THE TWO GROUPS WITH ATRIAL FIBRILLATION}

Group 1 patients were significantly $(p<0.001)$ older than those of group 2 (mean age 52 and 43 years, respectively), but there was no difference in the sex distribution. Patients in group 2 had used significantly larger amounts of alcohol daily during the week preceding atrial fibrillation than those in group $1\left(\chi^{2}=7.753 ; p<0.025\right)$ (fig 1$)$. In contrast there was no statistical difference between the groups for alcohol use in the two days before atrial fibrillation; $38 \%$ in group 1 and $54 \%$ in group 2 had used alcohol during that time.

Table 4 shows the frequencies of abnormal laboratory findings. There were no statistically significant differences between the groups for any of the laboratory measurements. Fifty per cent of cases in group 1 and $54 \%$ in group 2 who had drunk $>30 \mathrm{~g}$ of ethanol daily had one or more of the liver enzyme values above normal limits. Only one patient in the highest alcohol consumption category had increased mean red cell corpuscular volume. The abnormally low electrolyte values were evenly distributed between the groups and they were not related to the amount of alcohol used. 


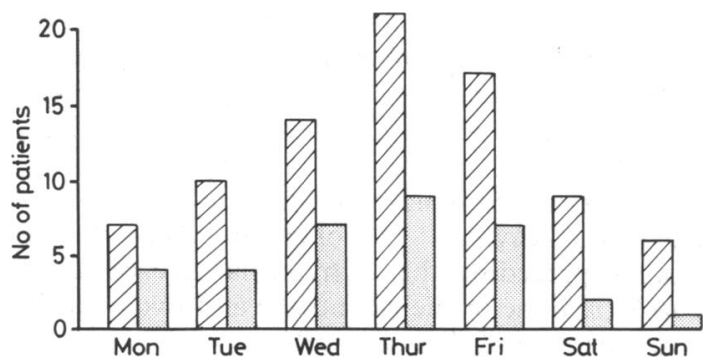

Fig 2 Distribution of the onset of atrial fibrillation in 84 patients by days of the week. The hatched columns indicate patients with atrial fibrillation and an associated disease and the stippled columns show patients with idiopathic atrial fibrillation.

VARIATION BY WEEKDAYS IN THE ONSET OF ATRIAL FIBRILLATION

Eighty four patients (50 in group 1 and 34 in group 2) were certain of the day on which arrhythmia had started. Figure 2 shows the day by day distribution of the onset of atrial fibrillation in both groups. The distribution differed significantly from a chance occurrence $\left(\chi^{2}=15.332 ; p<0.025\right)$ with the highest frequencies occurring in the middle of the week. In both groups 1 and 2 there was a similar trend but this did not quite reach statistical significance. Patients with high alcohol consumption showed a similar variation by weekday as did other cases. Fourteen $(70 \%)$ of those 20 patients who had consumed $>30$ $\mathrm{g}$ /day during the previous week had onset of arrhythmia on Wednesday, Thursday, or Friday.

\section{Discussion}

Taken together with earlier investigations, ${ }^{1-3}$ our study strongly supports the idea that alcohol plays a significant role in the genesis of otherwise inexplicable atrial fibrillation. The frequency of alcohol related atrial fibrillation, however, was clearly lower in our study than in two recent retrospective studies. ${ }^{12}$ Only one third of patients admitted for idiopathic atrial fibrillation had consumed more than $30 \mathrm{~g}$ of ethanol daily during the preceding week and not more than five of 35 patients had had more than $75 \mathrm{~g}$ a day. These data suggest that in about $15-30 \%$ of patients with idiopathic atrial fibrillation the arrhythmia may be alcohol related and that about $5-10 \%$ of all new episodes of atrial fibrillation can be explained by alcohol use. Among other identifiable diagnoses associated with new onset atrial fibrillation (table 2) alcohol abuse would thus rank above wellknown factors such as valvar heart disease, chronic obstructive pulmonary disease, and thyrotoxicosis. The true aetiological contribution of alcohol to atrial fibrillation may be somewhat larger because by causing dilated cardiomyopathy ${ }^{13}$ or myocardial infarction despite patent coronary arteries ${ }^{14}$ it can be the primary, although not the immediate, cause of the arrhythmia. Two of our four patients with dilated cardiomyopathy were alcoholics; they denied recent drinking. As well as being the main cause of atrial fibrillation in some patients, alcohol may be a triggering factor in patients with other heart diseases. This is suggested by our finding that a larger proportion of patients with aetiologically identifiable atrial fibrillation than of controls had used alcohol within the two days preceding the onset of the arrhythmia.

The selection of control patients in a hospital based study is always difficult and open to criticism. In our study, however, the alcohol consumption of the controls resembled that of a representative sample of 15-69 year old Finns whose alcohol use was studied in $1984 .{ }^{15}$ In that study 3870 people were questioned in detail about their drinking habits during one week and their alcohol use in general during the past year was evaluated. Twenty seven per cent of women and $12 \%$ of men had abstained in the previous year. The calculated mean yearly alcohol consumption (alcohol used during the week in question times 52) of the male drinkers was $4.1 \mathrm{~kg}$ (median $1.9 \mathrm{~kg}$ ) of ethanol and for female drinkers it was $1.1 \mathrm{~kg}$ (median $0.4 \mathrm{~kg}$ ). In our control group the frequencies of teetotallers $32 \%$ in women, $11 \%$ in men) were very close to those found in the population study. Mean yearly ethanol consumption of those controls who had drunk alcohol during the past year was $3.8 \mathrm{~kg}$ (median $1.5 \mathrm{~kg}$ ) in men and $0.2 \mathrm{~kg}$ (median 0 ) in women. The consumption of our male controls accords well with the population study whereas that of our female patients seems too small. There were only 13 drinkers among our female controls, however, and this may partly explain the discrepancy, especially as we evaluated only one week's alcohol intake. An important finding was that the patients with atrial fibrillation had drunk much larger quantities of ethanol: the mean values for yearly consumption were $9.1 \mathrm{~kg}$ (median $5.3 \mathrm{~kg}$ ) in male drinkers and $6.3 \mathrm{~kg}$ (median 0 ) in female drinkers. In our view these data support our conclusion that atrial fibrillation may be precipitated by a high intake of alcohol.

Probable mechanisms by which alcohol may induce atrial fibrillation have been discussed recently. ${ }^{15}$ Alcohol can increase the concentration of circulating catecholamines, ${ }^{16}$ change conduction times and refractory periods in the myocardium, ${ }^{4517}$ evoke sudden increases in vagal tone 
(vomiting), and produce subclinical myocardial injury when used heavily for many years. ${ }^{13}$ It may well be that in most cases a combination of two or more of these influences triggers the arrhythmia after binge drinking. It is our impression that factors such as physical or mental stress, lack of sleep, or febrile respiratory infections may at times increase the arrhythmogenic capacity of alcohol, probably by augmenting the basal sympathoadrenergic tone. In the present study electrolyte disturbances were not related to alcohol use and most patients had serum electrolytes within normal range on admission, although there were a surprising number with marginally lowered concentrations of serum total calcium. Unfortunately, serum albumin and ionised calcium were not measured, so there is no proof of real hypocalcaemia in these patients.

The term "holiday heart", introduced by Ettinger et $a l,{ }^{3}$ has been widely adopted to describe alcohol related arrhythmias clustering after the weekend and after holidays such as Christmas and New Year. In our study, however, the frequencies of the onset of atrial fibrillation were lowest during the weekend and in the days afterwards and highest in the middle of the week, irrespective of the aetiology of the arrhythmia. Nor did we see any excess of cases after festivals such as the New Year, May Day, or Midsummer Day which are all associated with alcohol sprees in Finland. In their retrospective study, Rich et al did not find clusters of admissions for alcohol related atrial fibrillation after weekends or holidays. ${ }^{2}$ It is possible that occupational mental or physical stress, which increases during the working week, together with other factors such as alcohol contribute to the onset of fibrillation.

Our study shows that ischaemic heart disease, hypertension, and cardiomyopathy are currently the most common diseases associated with new onset atrial fibrillation in young and middle aged population. Mitral valve disease and thyrotoxicosis, both still cited in textbooks as important causes of atrial fibrillation, now seem to be rare in this age group. About one third of patients presenting with their first episode of atrial fibrillation do not have any recognisable heart disease but many of them $(15-30 \%)$ are frequent drinkers and some drink quite heavily. There is no doubt that alcohol should be included among the most common aetiological factors causing atrial fibrillation in those of working age.

\section{References}

1 Lowenstein AJ, Gabow PA, Cramer J, Oliva PB, Ratner $K$. The role of alcohol in new-onset atrial fibrillation. Arch Intern Med 1983;143:1882-5.

2 Rich EC, Siebold C, Campion B. Alcohol-related acute atrial fibrillation. A case-control study and review of 40 patients. Arch Intern Med 1985;145:830-3.

3 Ettinger PO, Wu CF, De La Cruz C, Weisse AB, Ahmed SS, Regan TJ. Arrhythmias and the "holiday heart": alcohol-associated cardiac rhythm disorders. Am Heart J 1978;95:555-62.

4 Greenspon AJ, Shchaal SF. The "holiday heart": electrophysiologic studies of alcohol effects in alcoholics. Ann Intern Med 1983;98:135-9.

5 Engel TR, Luck JC. Effect of whisky on atrial vulnerability and "holiday heart". J Am Coll Cardiol 1983;1:816-8.

6 Thornton JR. Atrial fibrillation in healthy nonalcoholic people after an alcoholic binge. Lancet 1984;ii:1013-4.

7 Lamb LE, Pollard LW. Atrial fibrillation in flying personel. Report of 60 cases. Circulation 1964;29:694-701.

8 Peter RH, Gracey JG, Beach TB. A clinical profile of idiopathic atrial fibrillation. A functional disorder of atrial rhythm. Ann Intern Med 1968;68:1288-95.

9 Fleiss JL, Everitt BS. Comparing the marginal totals of square contingency tables. $\mathrm{Br} J$ Math Stat Psychol 1971;24:117-23.

10 Breslow NE, Day NE. Statistical methods in cancer research. Lyon: International Agency for Research on Cancer, 1980:182-8.

11 Huston TP, Puffer JC, Rodney WM. The athletic heart syndrome. N Engl J Med 1985;313:24-32.

12 Virtanen KS. Evidence of myocardial ischaemia in patients with chest pain syndromes and normal coronary angiograms. Acta Med Scand [Suppl] 1984;694:58-68.

13 Bridgen W, Robinson J. Alcoholic heart disease. $\mathrm{Br}$ Med J 1964;ii:1283-9.

14 Regan TJ, Wu CF, Weisse AB, Moschos CB, Ahmed SS, Lyons MM. Acute myocardial infarction in toxic cardiomyopathy without coronary obstruction. Circulation 1975;51:453-61.

15 Simpura J, ed. Finnish drinking habits. Results from interview surveys in 1968, 1976 and 1984. Helsinki: Finnish Foundation for Alcohol Studies, 1987.

16 Anonymous. Alcohol and atrial fibrillation. Lancet 1985;i:1374.

17 Perman ES. The effect of ethyl alcohol on the secretion from adrenal medulla in man. Acta Physiol Scand 1958;44:241-7.

18 Gould L, Reddy CVR, Becker W, Oh K-C, Kim SG. Electrophysiologic properties of alcohol in man. $J$ Electrocardiol 1978;11:219-26. 